\title{
Autoantibodies against AT1 Receptor Contribute to Vascular Aging and Endothelial Cell Senescence
}

\author{
Meili Wang ${ }^{1,2}$, Xiaochen Yin ${ }^{1,2}$, Suli Zhang, ${ }^{1,2}$, Chenfeng Mao ${ }^{3,4}$, Ning Cao ${ }^{1,2}$, Xiaochun Yang \\ Jingwei Bian ${ }^{1,2}$, Weiwei Hao ${ }^{1,2}$, Qian Fan ${ }^{5}$, Huirong Liu ${ }^{1,2}$ \\ ${ }^{1}$ Department of Physiology and Pathophysiology, School of Basic Medical Sciences, Capital Medical University, \\ Beijing, China. \\ ${ }^{2}$ Beijing Key Laboratory of Metabolic Disorders Related Cardiovascular Disease, Capital Medical University, \\ Beijing, China. \\ ${ }^{3}$ Department of Physiology and Pathophysiology, School of Basic Medical Sciences, Peking University Health \\ Science Center, Beijing, China. \\ ${ }^{4}$ Key Laboratory of Molecular Cardiovascular Science, Ministry of Education, Beijing, China. \\ ${ }^{5}$ Beijing Anzhen Hospital, Capital Medical University, Beijing, China.
}

[Received August 7, 2018; Revised September 15, 2018; Accepted September 19, 2018]

\begin{abstract}
Vascular aging predisposes the elderly to the progression of many aging-related vascular disorders and leads to deterioration of cardiovascular diseases (CVD). However, the underlying mechanisms have not been clearly elucidated. Agonistic autoantibodies against angiotensin II type 1 (AT1) receptor (AT1-AAs) have been demonstrated to be pro-inflammatory and contribute to the progression of atherosclerosis. However, the association between AT1-AAs and vascular aging has not been defined. Peripheral arterial disease (PAD) is an acknowledged vascular aging-related disease. In this study, AT1-AAs were detected in the sera of patients with PAD and the positive rate was $44.44 \%(n=63) v s .17 .46 \%$ in non-PAD volunteers $(n=63)$. In addition, case-control analysis showed that AT1-AAs level was positively correlated with PAD. To reveal the causal relationship between AT1-AAs and vascular aging, an AT1-AAs-positive rat model was established by active immunization. The carotid pulse wave velocity was higher, and the aortic endothelium-dependent vasodilatation was attenuated significantly in the immunized rats. Morphological staining showed thickening of the aortic wall. Histological examination showed that levels of the senescent markers were increased in the aortic tissue, mostly located at the endothelium. In addition, purified AT1-AAs-IgGs from both the immunized rats and PAD patients induced premature senescence in cultured human umbilical vein endothelial cells. These effects were significantly blocked by the AT1 receptor blocker. Taken together, our study demonstrates that AT1-AAs contribute to the progression of vascular aging and induce EC senescence through AT1 receptor. AT1-AA is a novel biomarker of vascular aging and aging-related CVD that acts to accelerate $\mathrm{EC}$ senescence.
\end{abstract}

Key words: AT1 receptor, Autoantibody, Peripheral arterial disease, Vascular aging, EC senescence

Vascular aging is a dominant risk factor for many agingrelated cardiovascular diseases (CVD), represented by atherosclerosis, coronary artery disease, vascular calcification, hypertension and stroke [1]. Many structural and functional changes are involved in this process, including endothelial dysfunction, vascular stiffening, increased secretion of inflammatory factors, and accumulation of oxidative stress [2]. Especially, vascular

*Correspondence should be addressed to: Dr. Huirong Liu, Department of Physiology and Pathophysiology, School of Basic Medical Sciences, Capital Medical University, No.10 Xitoutiao, You An Men, Beijing 100069, China. liuhr2000@ccmu.edu.cn

Copyright: () 2018 Wang M et al. This is an open-access article distributed under the terms of the Creative Commons Attribution License, which permits unrestricted use, distribution, and reproduction in any medium, provided the original author and source are credited. 
aging is characterized by endothelial dysfunction, which is associated with decreased endothelium-dependent relaxation during aging in humans [3]. Stiffening of large elastic arteries is recognized as another key vascular change associated with the development of endothelial dysfunction during aging $[4,5]$. Senescent vascular cells, particularly senescent endothelial cells (ECs), impair the vascular function and contribute to aging-related vascular diseases [6, 7]. But how genetic and environmental factors influence ECs and induce senescence remains largely unknown.

As a key regulator of vascular physiology, the reninangiotensin system (RAS) has been implicated in the development and progression of vascular aging [8]. Interruption of the RAS pathway, either by preventing the formation of angiotensin II (Ang II) or by blocking the Ang II type 1 (AT1) receptor, has been proven to be highly successful in retarding vascular aging phenotypes $[9,10]$. Meanwhile, inappropriate activation of the RAS, independent of the classic bioactive molecule Ang II, may cause excessive activation of the AT1 receptor and induce chronic inflammation $[11,12]$, but how this occurs is not fully understood.

At the end of the twentieth century, a specific autoantibody against AT1 receptor (AT1-AA) has been discovered and was found to exist in patients with preeclampsia [13], malignant hypertension [14], refractory hypertension [15] and renal-allograft rejection [16]. AT1-AAs could specifically bind to the second extracellular loop of AT1 receptor and were found to have a receptor agonist-like effect [13]. AT1-AAs were proven to be pro-inflammatory via the transcription factor nuclear factor-kappa B (NF-kB) pathway, thus enhancing the expression of inflammatory factors in ECs [17]. Moreover, we have previously demonstrated that AT1AAs induced endothelial damage and contributed to endothelial dysfunction in vivo [18]. Most importantly, AT1-AAs have been reported to accelerate aortic atherosclerosis in mice [19]. In a recent study, Peter M and colleagues demonstrated that higher AT1-AAs level was associated with inflammation, hypertension and adverse outcomes [20]. All the above evidence suggests a close relationship between AT1-AAs and vascular aging. Nevertheless, whether AT1-AAs can induce vascular aging or EC senescence has never been explored.

To address this issue, we performed a case-control study to explore the association between serum AT1-AAs levels and peripheral arterial disease (PAD). Next, we conducted in vivo experiments with the use of AT1-AAspositive rat models to explore the role of AT1-AAs in inducing vascular aging. Further, we performed in vitro experiments to investigate the effect of AT1-AAs on EC senescence and the underlying mechanism.

\section{MATERIALS AND METHODS}

\section{Materials}

Antibodies against p53, p21, p16 $6^{\mathrm{INK} 4 \mathrm{a}}$ and $\beta$-actin were purchased from Abcam (Cambridge, UK). Antibodies against p-eNOS and eNOS were purchased from Cell Signaling Technology (Boston, MA, USA).

\section{Study population}

This study included a total of 126 participants, who were divided into 2 groups: One group was composed of 63 patients with PAD and the other was composed of 63 nonPAD volunteers. The serum samples of both groups were from Beijing Anzhen hospital. PAD was detected by the measurement of ankle-brachial index (ABI) and diagnosed by the presence of an ABI $<0.9$. Non-PAD volunteers with a negative medical history of PAD $(1.0<$ $\mathrm{ABI}<1.3)$ and no evidence of sonographically detectable atherosclerotic diseases served as controls.

This study was approved by the local research ethics committee (Beijing Anzhen hospital, Capital Medical University, Beijing, China). Written informed consents were obtained from all the participants before the study commenced.

\section{Animals}

Healthy male Sprague-Dawley (SD) rats aged 8 weeks (140-160g) were obtained from the Animal Center of Capital Medical University to establish the active immunization model. All the animal experiments followed the guidelines of the Institutional Animal Care and Use Committee and Ethics Committee of Capital Medical University.

\section{Active immunization}

Male SD rats were randomly divided into two groups: the immunized group and the Freund's adjuvant-treated vehicle group. The synthetic peptides corresponding to the sequence of the second extracellular loop of human AT1 receptor $\left(\mathrm{AT}_{1} \mathrm{R}-\mathrm{EC}_{\mathrm{II}}\right.$, residues 165-191, sequence IH-R-N-V-F-F-I-E-N-T-N-I-T-V-C-A-F-H-Y-E-S-Q-NS-T-L, 95\% purity, GL Biochem Ltd, Shanghai) were dissolved in $\mathrm{Na}_{2} \mathrm{CO}_{3}$ solution (100 mM, PH 11.0) to a final concentration of $4 \mathrm{mg} / \mathrm{ml}$ and then diluted in normal saline. The antigen solution, together with Freund's complete adjuvant by an equal proportion, was emulsified and then multiply-injected into the back of the rats subcutaneously $(0.4 \mathrm{mg} / \mathrm{kg})$. Booster immunizations were repeated once every two weeks by a single subcutaneous injection, and the antigen was emulsified in Freund's 
incomplete adjuvant. The antigen solution was replaced with $\mathrm{Na}_{2} \mathrm{CO}_{3}$ solution following the same procedure in the vehicle group. The blood samples extracted from the tail top of the rats were collected for detection of serum AT1AAs.

\section{Enzyme-linked immunosorbent assay (ELISA)}

Modified ELISA was used to detect the titers of AT1-AAs in sera as previously described [18]. The results were expressed as OD values measured at $405 \mathrm{~nm}$ using a Spectra Max Plus microplate reader (Molecular Devices Corp, Sunnyvale, USA). The positive/negative $(\mathrm{P} / \mathrm{N})$ ratio was calculated as follows: (OD of sample - OD of blank control)/(OD of negative control - OD of blank control). Samples with a $\mathrm{P} / \mathrm{N}$ value $>2.1$ was considered positive.

\section{Measurement of pulsed wave velocity $(P W V)$}

The rats were anesthetized with isoflurane and maintained by mask ventilation (3\% for induction and $1.5 \%$ for maintenance) with a coupled charcoal scavenging system during the measurement. PWV was measured at the distal and proximal locations of the left common carotid artery using a $15-\mathrm{MHz}$ Doppler probe while simultaneously recording the electrocardiogram (ECG) signal. PWV was calculated as a quotient of the separation distance and the transit time between pulse arrivals which was measured from the ECG R-peaks.

\section{Aortic ring vasodilatory response}

As the immunization process finished, rats were anesthetized intraperitoneally with pentobarbital sodium $(40 \mathrm{mg} / \mathrm{kg})$. The aorta was isolated from the rats of both vehicle and immunized groups. The dissected vessels were immediately removed and placed in ice-cold KrebsHenseleit buffer, and then cleaned of additional connective tissues carefully. The aorta was cut into 3-4 $\mathrm{mm}$ rings and subsequently subjected to vascular tension experiments. The changes in isometric force were recorded using a PowerLab system (AD Instruments, Australia). The rings were preconstricted with phenylephrine $(1 \mu \mathrm{M})$. After the contraction reached a plateau, cumulative doses of acetylcholine (ACh, 10-9$10^{-6} \mathrm{M}$ ) were added to characterize vasorelaxation. To investigate the role of endothelial nitric oxide synthase (eNOS), N-nitro-L-arginine-methyl ester (L-NAME, 100 $\mu \mathrm{M})$ was used for pretreatment, followed by the continuous addition of sodium nitroprusside (SNP, $10^{-9}$ $\left.10^{-6} \mathrm{M}\right)$. Vasorelaxation was presented as the percent relaxation, as calculated by the percent decrease in the tension from the phenylephrine-induced preconstriction.

\section{Preparation of the immunoglobulin $G$}

The blood samples were collected from the abdominal aorta of rats after completion of the immunization process. Total IgGs in the sera of both the rats and human were purified by IgG affinity column (Mab Trap Kit, Amersham) as previously described [18].

\section{Cell culture and treatment}

Human umbilical vein endothelial cells (HUVECs) were purchased from Shanghai Cell Bank, Chinese Academy of Sciences as pooled primary cell lines and cultured with endothelial growth medium (M199, Gibco, San Diego, CA) supplemented with $10 \%$ fetal bovine serum (FBS, HyClone, Logan, UT) at $37^{\circ} \mathrm{C}$ in a $5 \% \mathrm{CO}_{2}$ atmosphere. HUVECs were plated and cultured to 80 90\% confluence and treated with various concentrations $(0,0.001,0.01$, $0.1,1$ and $10 \mu \mathrm{M})$ of AT1-AAs for 5 days, or a concentration of $1 \mu \mathrm{M}$ of AT1-AAs, nIgGs, or Ang II for 0, 24, 48 and $72 \mathrm{hrs.} \mathrm{In} \mathrm{the} \mathrm{AT1} \mathrm{receptor} \mathrm{blockage} \mathrm{study,}$ similarly prepared HUVECs were incubated with valsartan $(10 \mu \mathrm{M})$ for 2 hrs prior to stimulation with AT1AAs for $72 \mathrm{hrs}$.

\section{Cell cycle assay}

The HUVECs were digested with trypsin, collected by centrifugation and fixed with $70 \%$ ethanol at $4{ }^{\circ} \mathrm{C}$ overnight. Then cells were re-suspended in PBS containing $50 \mathrm{mM}$ propidium iodide and $10 \mu \mathrm{g} / \mathrm{ml}$ RNase for 30 mins at $37^{\circ} \mathrm{C}$. After washing with cold PBS, cell cycles were analyzed by flow cytometric analysis (BD FACS Calibur, Becton Dickinson, East Rutherford, NJ, USA). The number of cells within the G0/G1, S and G2/M phases of the cell cycle was analyzed using ModFit LT, version 2.0 (Verify Software House, Topsham, ME).

\section{Senescence-associated $\beta$-galactosidase (SA $\beta$-Gal) assay}

The SA $\beta$-Gal staining was performed according to the manufacturer's instructions (Beyotime, China). Briefly, the HUVECs were washed with PBS and fixed with the fixative solution for $15 \mathrm{mins}$, and then washed and incubated with the staining solution at $37{ }^{\circ} \mathrm{C}$ for $16 \mathrm{hrs}$. Cells were counterstained with DAPI to count the total number and the senescent cells were blue-stained as observed under the light microscope (Olympus, Japan). The SA $\beta$-Gal positive cells were counted manually, and the total cell number was quantified using NIH ImageJ software (National Institutes of Health, USA). More than 30,000 cells total were counted in 9 randomly selected fields to determine the percentage of SA $\beta$-Gal-positive cells. 


\section{Immunohistochemistry}

Tissue sections were prepared and incubated with primary antibodies against p53 (at a dilution of 1:500), p21 (at a dilution of $1: 1000$ ) and $\mathrm{p} 16^{\mathrm{INK4a}}$ (at a dilution of 1:1000) overnight at $4{ }^{\circ} \mathrm{C}$. HRP-labeled anti-rabbit or anti-mouse antibodies were applied for $1 \mathrm{hr}$ at room temperature and cell nuclei were visualized with DAPI staining.

\section{Western blotting}

Protein concentrations were evaluated using a BCA Protein Assay Kit (Thermo Scientific, Waltham, MA). Whole cell lysates containing equal amounts of total protein $(25 \mu \mathrm{g})$ were separated by $8 \%$ 15\% SDS-PAGE gels and transferred onto PVDF membranes (Millipore, Billerica, MA). The membranes were blocked with 5\% milk (in TBST) for $1 \mathrm{hr}$, and then incubated with corresponding primary antibodies at $4{ }^{\circ} \mathrm{C}$ overnight. After washing, the membranes were incubated with secondary antibodies at a 1:20000 dilution and then developed using an ECL regent (Applygen Technologies Inc.).

\section{$R T-q P C R$}

Total RNA was extracted from cultured HUVECs using the Trizol reagent (Invitrogen, USA), and an equal amount $(2 \mu \mathrm{g})$ was reverse transcribed into cDNA with the Reverse Transcription System. Quantitation of all gene transcripts was done by qPCR using Power SYBR Green PCR Master Mix (Thermo Scientific, Waltham, MA) and ABI PRISM 7500 sequence detection system (Applied Biosystems, Foster City, CA) with the expression of GAPDH as the internal control. Relative mRNA levels were presented as mean fold change of samples as compared with the control. The primers used are listed in Supplementary Table.

\section{Statistical methods}

All the animal experiments and cell assay data were expressed as mean \pm SEM. Differences between two groups were analyzed using Student's $t$-test. One-way ANOVA followed by Student-Newman-Keuls test was used in pairwise comparison of three or more groups. Chisquare test was used in comparison of rate between two groups. GraphPad Prism 6.0 (GraphPad Software, Inc., San Diego, CA, USA) was used for drafting and statistical analysis; $p$ values $<0.05$ were considered statistically significant.

Table 1. Chi-square analysis on demographic characteristics of participants.

\begin{tabular}{|c|c|c|c|c|c|}
\hline \multirow{2}{*}{ Characteristic } & & \multicolumn{2}{|c|}{ PAD } & \multirow{2}{*}{$\chi^{2}$} & \multirow{2}{*}{$\mathbf{P}$} \\
\hline & & Yes $(\%)$ & No (\%) & & \\
\hline \multirow{2}{*}{ Gender $^{\triangle}$} & male & $46(73.02)$ & $46(73.02)$ & \multirow{2}{*}{ - } & \multirow{2}{*}{ - } \\
\hline & female & $17(26.98)$ & $17(26.98)$ & & \\
\hline \multirow{3}{*}{ Smoking history } & Ever & $16(25.40)$ & $10(15.87)$ & \multirow{3}{*}{16.391} & \multirow{3}{*}{$<0.001$} \\
\hline & Current & $34(53.97)$ & $18(28.57)$ & & \\
\hline & Never & $13(20.63)$ & $35(55.56)$ & & \\
\hline \multirow{3}{*}{ Drinking history } & Ever & $9(14.29)$ & $10(15.87)$ & \multirow{3}{*}{0.184} & \multirow{3}{*}{0.912} \\
\hline & Current & $18(28.57)$ & $16(25.40)$ & & \\
\hline & Never & $36(57.14)$ & $37(58.73)$ & & \\
\hline \multirow{3}{*}{ Hypertension history } & No & $15(23.81)$ & $32(50.79)$ & \multirow{3}{*}{11.097} & \multirow{3}{*}{0.004} \\
\hline & Yes, good drug control & $25(39.68)$ & $12(19.05)$ & & \\
\hline & Yes, bad drug control & $23(36.51)$ & $19(30.16)$ & & \\
\hline \multirow{2}{*}{ Diabetes } & No & $42(66.67)$ & $60(95.24)$ & \multirow{2}{*}{16.676} & \multirow{2}{*}{$<0.001$} \\
\hline & Yes & $21(33.33)$ & $3(4.76)$ & & \\
\hline
\end{tabular}

${ }^{\triangle}$ Matching factors; PAD, peripheral arterial disease

\section{RESULTS}

\section{Detection of AT1-AAs in patients with PAD}

Epidemiological studies have shown that PAD, mainly characterized by the presence of increased arterial stiffness, is prevalent in the elderly. Clinically, PAD confers an independent risk for cardiovascular events and total mortality [21, 22]. To assess the association of AT1-
AAs with vascular aging-related diseases, we collected sera from 63 PAD patients recruited from Beijing Anzhen hospital and determined the level of AT1-AAs. As shown in Fig. 1A, AT1-AAs were identified as positive in 28 patients, which was significantly higher compared to the non-PAD volunteers as control group (PAD: $44.44 \%$ vs CTL: $17.46 \%, \mathrm{n}=63$ per group, $\mathrm{p}<0.001)$. In addition, the levels of AT1-AAs in PAD patients were generally higher than these in non-PAD volunteers (Fig. 1B). 
A

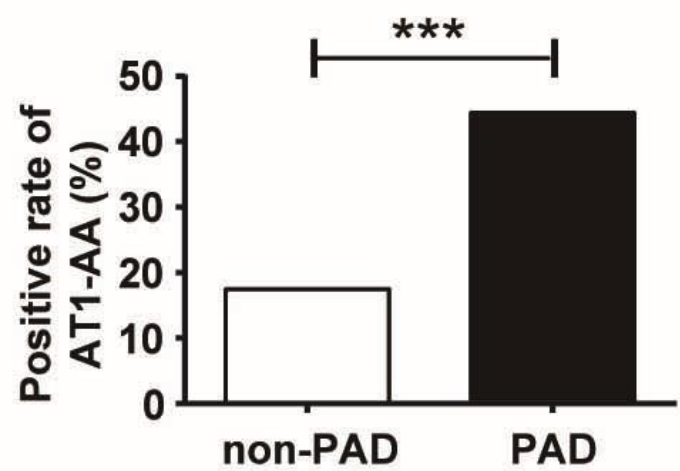

B

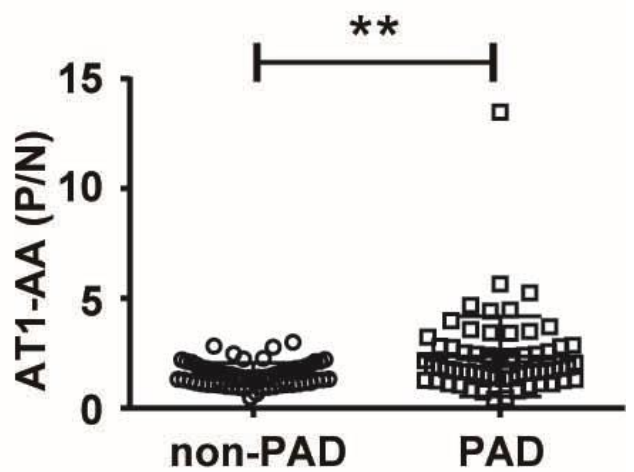

Figure 1. High levels of AT1-AAs in the patients with PAD. A) The positive rate of AT1-AAs in PAD group and the nonPAD group was $44.44 \%$ and $17.46 \%$, respectively. ( $n=63$ per group). B) The P/N values of AT1-AAs in the sera samples of PAD patients and non-PAD participants measured by ELISA. PAD, peripheral arterial disease, $* * p<0.01, * * * p<0.001$.

\section{Univariate analysis on participants' characteristics}

A hospital based 1:1 matched case-control study was carried out, involving a total of 126 participants. A pair was composed of one PAD patient and one gender- and age-matched non-PAD volunteer. As shown in Table 1, cases (PAD patients) and controls (non-PAD volunteers) did not differ significantly with respect to the proportion whether drinking or not. However, the cases were significantly more likely to have a smoking history, as well as previous clinically diagnosed hypertension and diabetes history.

\section{Univariate analysis on the biochemical indexes of the participants}

Table 2 shows the results of Wilcoxon rank sum test on the biochemical indexes of all the participants. There was no significant difference in body mass index (BMI), heart rate, systolic blood pressure (SBP), diastolic blood pressure (DBP), blood sugar and high density lipoprotein (HDL) level between the two groups. However, the serum levels of AT1-AAs, total cholesterol (TC), triglyceride (TG) and low-density lipoprotein (LDL) in the cases were significantly higher than those in the control group.
A

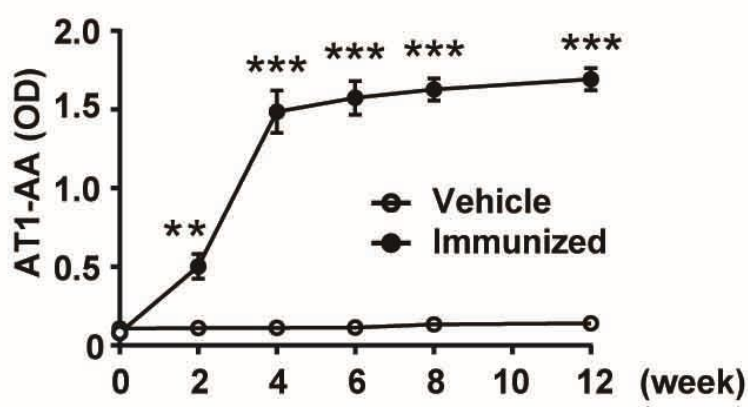

B

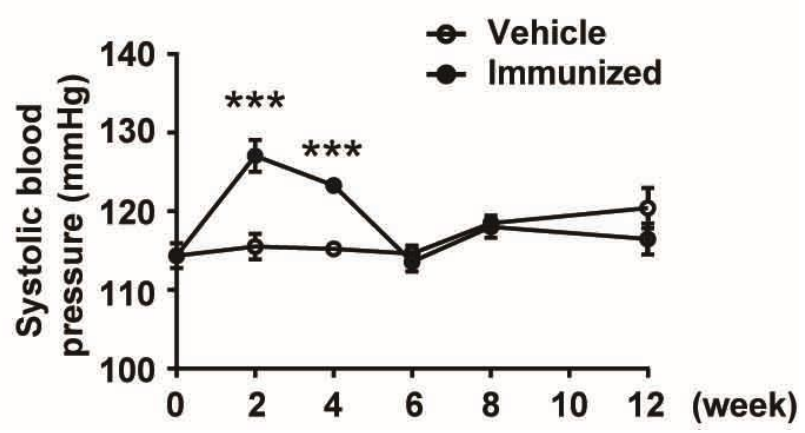

Figure 2. Successful establishment of the AT1-AAs-positive rat model. A) The level of AT1-AAs in sera was detected by ELISA. It increased gradually after the first immunization and reached a peak six weeks later and maintained a high value compared with the vehicle group. B) Blood pressure curves of rats immunized with $\mathrm{AT}_{1} \mathrm{R}-\mathrm{EC}$ II peptides or the vehicle group. Data were expressed as means \pm SEM, $\mathrm{n}=8-10$ rats per group. $* * * p<0.001 v s$. the vehicle group.

\section{Multivariate analysis of the entire study population}

As shown in Table 3, the serum level of AT1-AAs was significantly associated with PAD after adjustment for age, gender, smoking history, diabetes history, hypertension history, TC, TG and LDL levels. The risk for PAD in participants with high levels of AT1-AAs was significantly higher than that in those with low serum levels of AT1-AAs. Specifically, every one unit increase in the $\mathrm{P} / \mathrm{N}$ value increased the risk of PAD by 3.559 -fold $(\mathrm{OR}=3.559,95 \%$ C.I: $1.638,7.730)$. 
Table 2. Wilcoxon rank sum test on the biochemical indexes of PAD patients.

\begin{tabular}{lcccc}
\hline \multicolumn{1}{c}{ Variables } & PAD $(\mathbf{n}=\mathbf{6 3})$ & Non-PAD $(\mathbf{n}=\mathbf{6 3})$ & $\boldsymbol{Z}$ value & $\boldsymbol{P}$ value \\
\hline Age $\triangle$ & $65.29 \pm 13.12$ & $64.81 \pm 12.94$ & - & - \\
AT1-AA (P/N) & $2.362 \pm 1.828$ & $1.533 \pm 0.5299$ & -4.073 & $<0.001$ \\
BMI & $23.72 \pm 3.48$ & $24.69 \pm 3.784$ & -1.506 & 0.132 \\
Heart rate & $80.03 \pm 10.72$ & $77.46 \pm 13.04$ & -1.347 & 0.178 \\
SBP & $146.3 \pm 19.32$ & $142.7 \pm 17.35$ & -1.412 & 0.158 \\
DBP & $78.78 \pm 9.868$ & $82.84 \pm 13.89$ & -1.489 & 0.136 \\
Blood sugar level & $5.529 \pm 2.396$ & $5.539 \pm 1.232$ & -1.629 & 0.103 \\
TC (mmol/L) & $4.608 \pm 1.168$ & $4.171 \pm 1.070$ & -2.119 & 0.034 \\
TG (mmol/L) & $1.523 \pm 0.8398$ & $1.477 \pm 1.975$ & -2.499 & 0.012 \\
HDL (mmol/L) & $1.077 \pm 0.2667$ & $1.083 \pm 0.2648$ & -0.246 & 0.805 \\
LDL (mmol/L) & $2.956 \pm 0.9662$ & $2.582 \pm 0.7198$ & -2.341 & 0.019 \\
\hline Matching factors; PAD, peripheral arterial disease; BMI, Body Mass Index; TC, Total cholesterol; TG, Triglyceride; HDL, \\
high density lipoprotein; LDL, low density lipoprotein
\end{tabular}

\section{Successful establishment of AT1-AAs-positive rat models by active immunization}

After demonstrating the close association between the serum level of AT1-AAs and vascular aging-related disease, we sought to determine whether AT1-AAs could induce vascular aging. An AT1-AAs-positive rat model was established by active immunization with the synthetic peptides corresponding to the sequence of the second extracellular loop of human AT1 receptor $\left(\mathrm{AT}_{1} \mathrm{R}-\mathrm{EC}_{\mathrm{II}}\right)$. Since the initial immunization, the titers of AT1-AAs in the sera of rats were detected by using the enzyme-linked immunosorbent assay (ELISA) method. As illustrated in Fig. 2A, the serum level of AT1-AAs began increasing from the 2nd week and the concentration of AT1-AAs maintained high until the end of immunization, indicating the successful establishment of the active immunization model. Meanwhile, AT1-AAs were not detected in the vehicle group throughout the entire process. Additionally, we measured the SBP. It was found that SBP began increasing from the 2 nd week, remained high until the 4 th week, but showed no difference from the 6 th week to the end of the experiment (Fig. 2B).

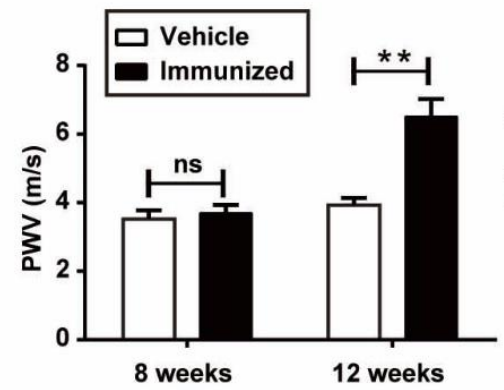

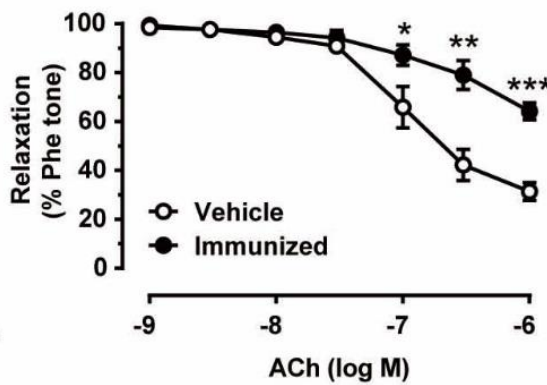

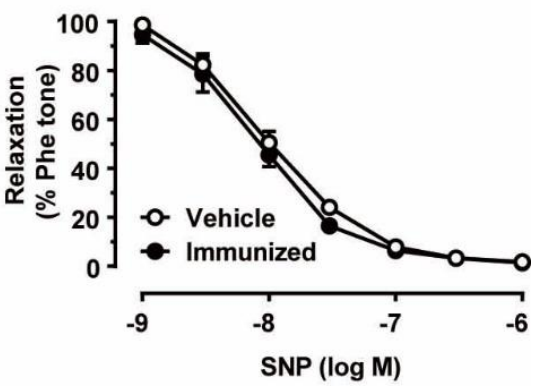

Figure 3. Functional evidence of vascular aging in immunized rats. A) Pulse wave velocity measured in the left common carotid artery from the vehicle and $\mathrm{AT}_{1} \mathrm{R}-\mathrm{EC}_{\mathrm{II}}$-immunized rats. B) Relaxation curves in response to the vasodilator acetylcholine of phenylephrine $(1 \mu \mathrm{M})$-pretreated isolated aortic rings from the vehicle and $\mathrm{AT}_{1} \mathrm{R}-\mathrm{EC}_{\mathrm{II}}$-immunized rats. C) Relaxation in response to SNP. Isolated aortic rings were treated with L-NAME $(100 \mu \mathrm{M})$ for 30 mins followed by phenylephrine $(1 \mu \mathrm{M})$ treatment. Data were expressed as mean \pm SEM. $\mathrm{n}=6-8$ rats for each group, ${ }^{*} p<0.05,{ }^{*} p<0.01, * * * p<0.001 v s$. the vehicle group.

\section{AT1-AAs caused vascular aging and endothelial senescence in actively immunized rats}

Vascular stiffening is the hallmark of vascular aging, and PWV is considered the gold standard measure of vascular stiffness in humans [23]. In this study, PWV of the rats was measured noninvasively in the left common carotid artery (Supplementary Fig. 1). To determine the earliest timepoint of the occurance of changes in the vascular physiological parameters, we measured the PWV at the $8^{\text {th }}$ and $12^{\text {th }}$ weeks after initial immunization. As shown in Fig. 3A, there was no significant difference in PWV 
between the vehicle and $\mathrm{AT}_{1} \mathrm{R}-\mathrm{EC}_{\mathrm{II}}$-immunized groups at the $8^{\text {th }}$ week. However, PWV was increased at the $12^{\text {th }}$ week. To further investigate the association between AT1-AAs and vascular aging, an isometric tension experiment was conducted to evaluate the vasodilatation of the aortas. The aortic rings obtained from the two groups were pre-contracted with $1 \mu \mathrm{M}$ phenylephrine and then treated with acetylcholine $(\mathrm{ACh})\left(10^{-9}-10^{-6} \mathrm{M}\right)$. As summarized in Fig. 3B, compared with the vehicle group, reduced ACh-induced vasodilatation was observed in the immunized group. Meanwhile, the phosphorylation of endothelial nitric oxide synthase (eNOS) was reduced in the aortic tissues of the immunized rats (Supplementary Fig. 2). These results suggested that AT1-AAs induced dysfunction of endothelium-dependent vasodilatation. Nevertheless, there was no significant difference in sodium nitroprusside (SNP) $\left(10^{-9}-10^{-6} \quad \mathrm{M}\right)$-induced endothelium-independent vasodilatation between the two groups (Fig. 3C). These data demonstrated that AT1-AAs led to vascular aging-related dysfunction in vivo.
A

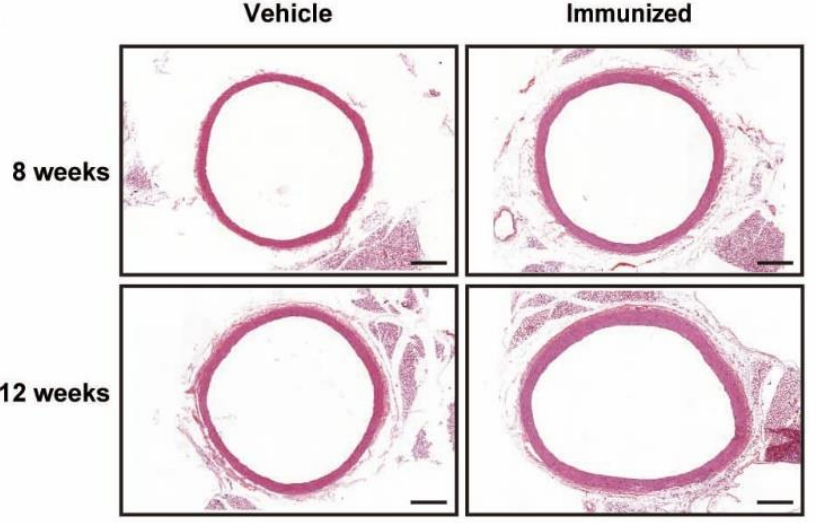

B

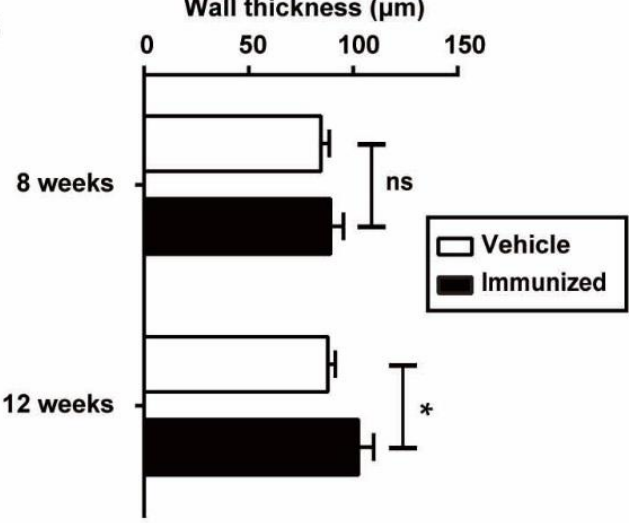

C

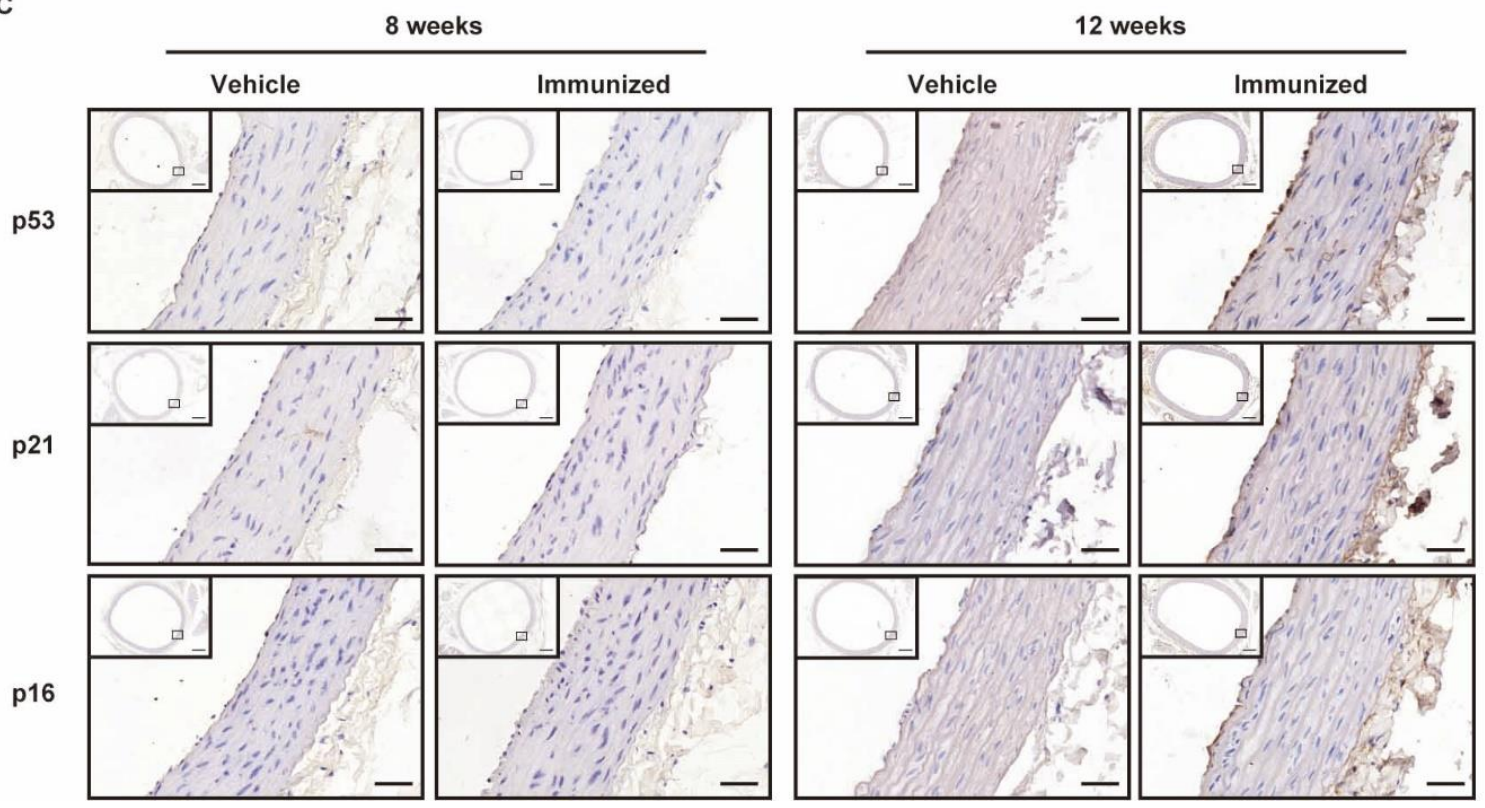

Figure 4. Changes in arterial morphology after immunization. A) Microscopic images of the HE-stained aortic sections. Scale bar $=400 \mu \mathrm{m}$. There was no significant difference in arterial wall thickness at the $8^{\text {th }}$ weeks, but it increased significantly after 12-week immunization. B) Quantitative analysis of wall thickness of thoracic aortas at 8 and 12 weeks after immunization. C) Representative images of immunohistochemical staining showing p53, p21 and p16 ${ }^{\text {INK4a }}$ in the rat aorta of the indicated groups. Scale bar $=400$ (small views) and $40 \mu \mathrm{m}$ (enlarged views). Data were expressed as mean \pm SEM. $\mathrm{n}=6-8$ rats for each group, ${ }^{*} p<0.05$. 


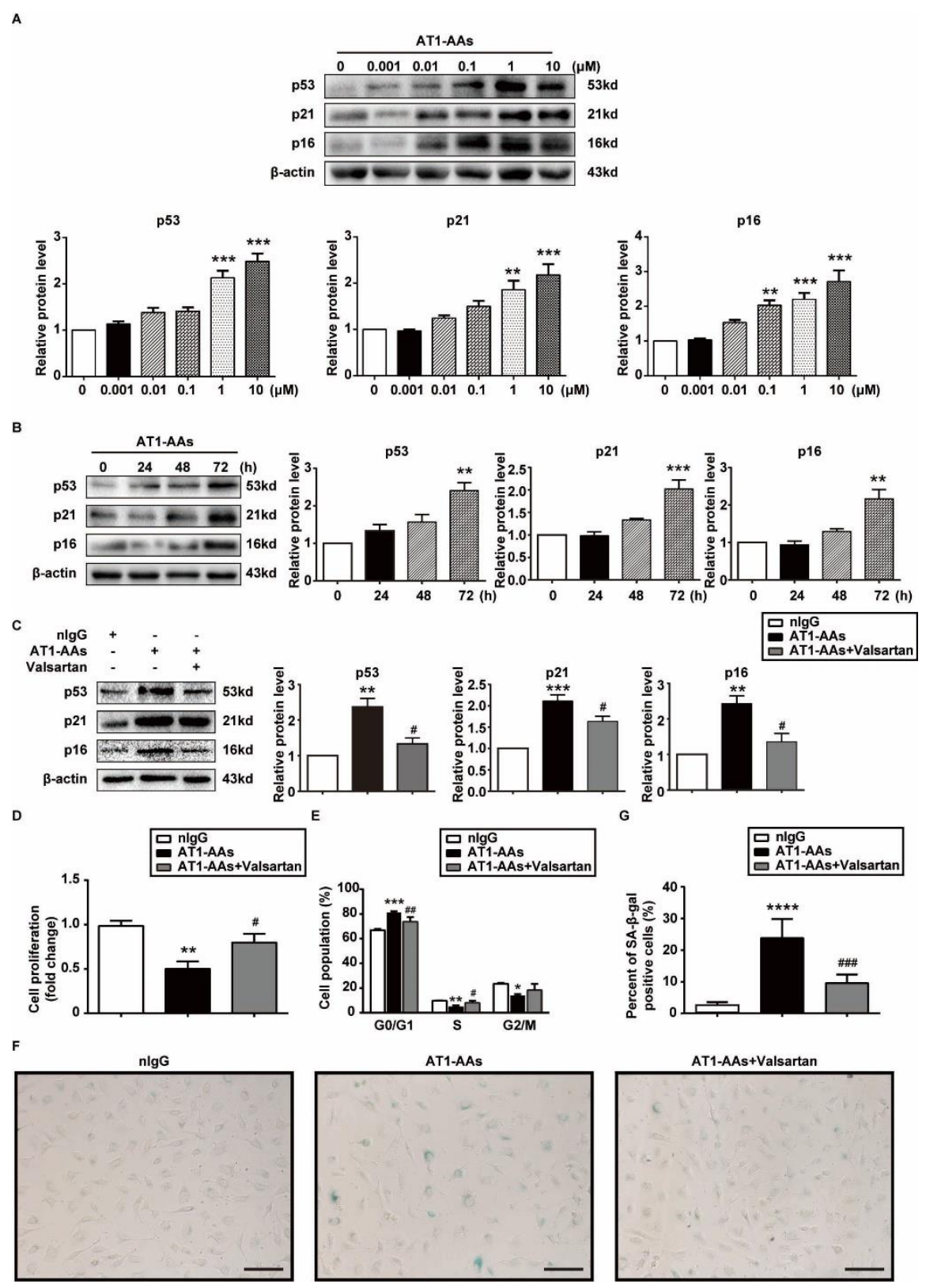

Figure 5. AT1-AAs induced premature senescence of HUVECs through AT1 receptor. A) Representative images and quantitative graphs of p53, p21 and p16 $6^{\mathrm{INK} 4 \mathrm{a}}$ expressions. HUVECs were incubated with AT1-AAs-IgGs of indicated concentrations for 5 days. B) Representative images and quantitative graphs of p53, p21 and p16 ${ }^{\mathrm{INK} 4 \mathrm{a}}$ expressions. HUVECs were incubated with AT1-AAs-IgGs $(1 \mu \mathrm{M})$ for indicated times. ${ }^{* *} p<0.01$, $* * * p<0.001 v s$. the control group. C) Representative western blot and quantitative graphs of p53, p21 and p16 ${ }^{\mathrm{INK} 4 \mathrm{a}}$ expressions in HUVECs treated with nIgGs, AT1-AA or valsartan plus AT1-AA for 72 hrs. D. Measurement of cell proliferation using the cck- 8 analysis. HUVECs treated with AT1-AA manifested a reduction in cell proliferation by comparison to the $\mathrm{nIgG}$ treated group. E) Cell cycle analysis of the nIgGs, AT1-AAs-IgGs or valsartan plus AT1-AAs-IgGs-induced HUVECs by flow cytometry. F) Photographs of typical SA- $\beta$ gal-stained HUVECs in the nIgGs, AT1-AA and valsartan+AT1-AA groups (senescent cells are stained blue). Scale bar $=400 \mu \mathrm{m}$. H. Quantification of percentages of SA- $\beta$-gal-positive HUVECs of the indicated groups. Data in the graphs were from 3 independent experiments and were expressed as mean \pm SEM. $* p<0.05, * * p<0.01, * * * p<0.001,{ }^{*} * * * p<0.0001 v s$. the nIgGs group; \# $p<0.05$, \#\# $p<0.01$, \#\#\# $p<0.001 v s$. the AT1-AAs-IgGs group. 
To clarify the pathological changes and characterize the progression of vascular aging, the aortas were harvested and subjected to hematoxylin-eosin staining. Significant thickening of the aortic wall was observed (Fig. 4A, B). Moreover, immunohistochemical staining for $\mathrm{p} 53, \mathrm{p} 21$ and $\mathrm{p} 16^{\mathrm{INK} 4 \mathrm{a}}$ showed that AT1-AAs markedly enhanced expression of these aging-related molecules at the 12th week. Notably, the prominent increase was mainly distributed in the endothelial layer (Fig. 4C). Altogether, these results provided direct evidence that AT1-AAs caused vascular aging in vivo.

\section{AT1-AAs induced human umbilical vein endothelial cells (HUVECs) senescence via AT1 receptor}

The total IgGs extracted from the immunized rats (termed AT1-AAs-IgGs) were loaded onto the sodium dodecyl sulfate polyacrylamide gel electrophoresis and then processed with Coomassie brilliant blue staining. The results showed that two strong bands were seen at $55 \mathrm{kDa}$ and $25 \mathrm{kDa}$, representing the heavy and light chain of total IgGs, respectively (Supplementary Fig. 3A). To determine the activity of AT1-AAs-IgGs, the beating frequency of neonatal rat cardiomyocytes (NRCMs) was counted. As shown in Supplementary Fig. 3B, the AT1AAs-IgGs strongly enhanced the beating frequency of NRCMs, which was inhibited by the AT1 receptor blocker, valsartan.

A
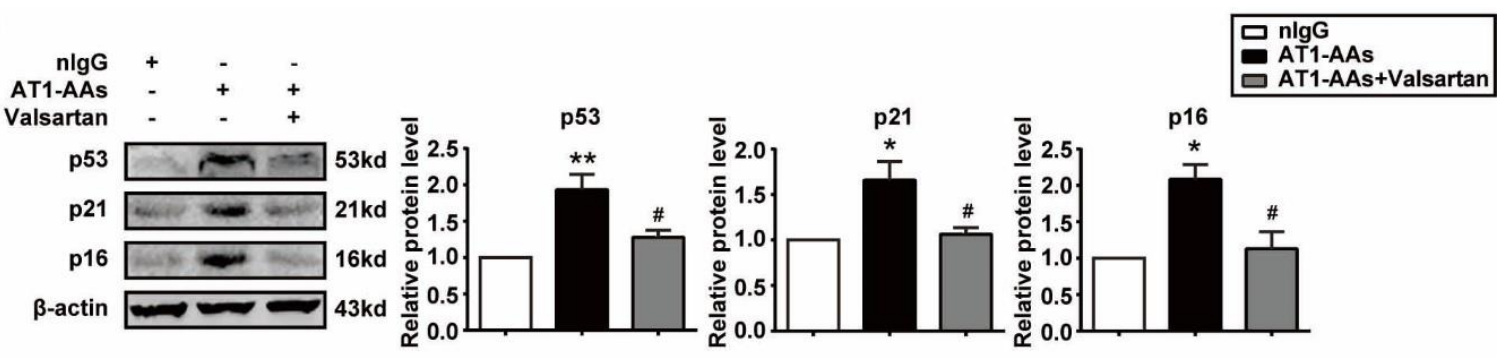

B
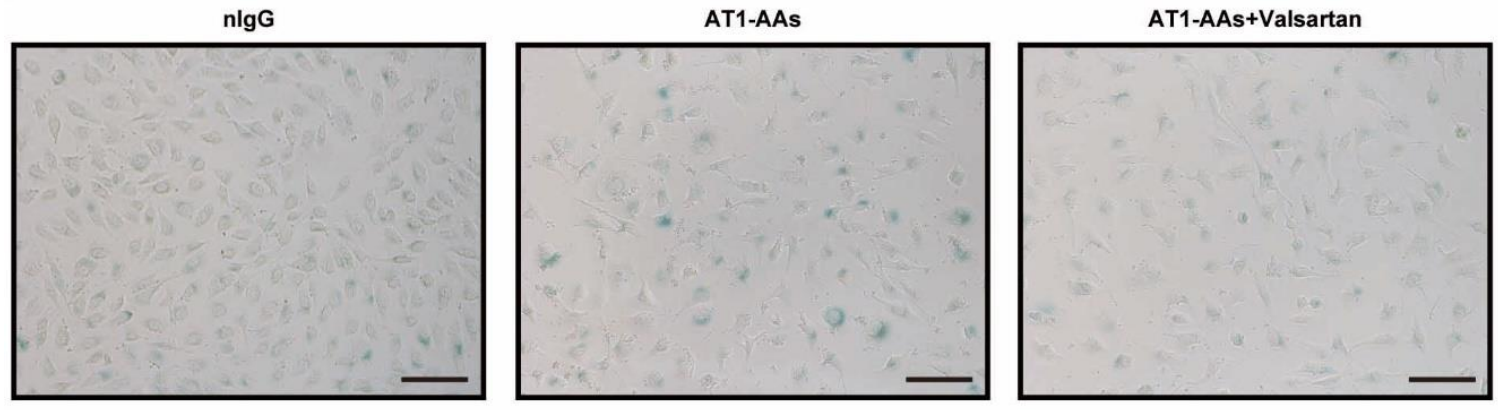

C

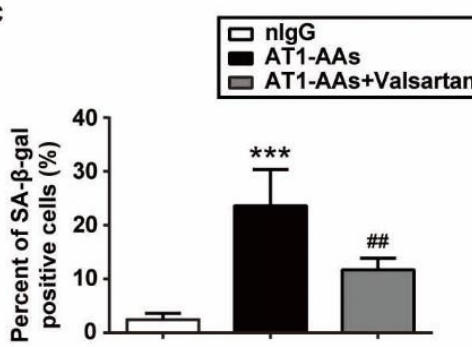

Figure 6. AT1-AAs from the PAD patients induced HUVECs senescence. A) Representative western blot and quantitative graphs of p53, p21 and p16 ${ }^{\mathrm{INK} 4 \mathrm{a}}$ expressions in HUVECs treated with $\mathrm{nIgGs}$, AT1-AAs-IgGs or valsartan plus AT1-AAs-IgGs for 72 hrs. B) Photographs of typical SA- $\beta$-gal-stained HUVECs in the nIgGs, AT1-AAs-IgGs and valsartan+AT1-AAs-IgGs groups. Scale bar $=400 \mu \mathrm{m}$. C) Quantification of percentages of SA- $\beta$-gal-positive HUVECs of the indicated groups. Data in the graphs were from 3 independent experiments and were expressed as mean \pm SEM. $* p$ $<0.05,{ }^{*} p<0.01, * * * p<0.001 v s$. the nIgGs group; \# $p<0.05$, \#\# $p<0.01$ vs. the AT1-AAs group. 
Table 3. Logistic regression analysis.

\begin{tabular}{lllllll}
\hline Variables & B & Wald value & $\boldsymbol{P}$ value & OR & 95\%C.I. for OR \\
\hline Constant & -4.561 & 5.501 & 0.019 & 0.010 & & \\
AT1-AA $(\mathrm{P} / \mathrm{N})$ & 1.269 & 10.284 & 0.001 & 3.559 & 1.638 & 7.730 \\
\hline
\end{tabular}

$\mathrm{B}$, partial regression coefficient; OR, odds ratio; C.1., confidence interval

To further investigate the association between AT1AAs and vascular aging and vascular cells senescence, in vitro experiments were conducted with HUVECs. HUVECs were incubated with different concentrations ( 0 , $0.001,0.01,0.1,1$ and $10 \mu \mathrm{M})$ of AT1-AAs-IgGs for 5 days. As shown in Fig. 5A, protein levels of p53, p21 and $\mathrm{p} 16^{\mathrm{INK} 4 \mathrm{a}}$ were all spontaneously and persistently increased in cells treated with $1 \mu \mathrm{M}$ of AT1-AAs-IgGs. To further explore the effect of AT1-AAs on these proteins, HUVECs were treated with AT1-AAs $(1 \mu \mathrm{M})$ for various time intervals. The time-course experiment showed that AT1-AAs triggered a significant up-regulation of these senescent markers at $72 \mathrm{hrs}$ (Fig. 5B). In contrast, no significant change was observed in the negative $\operatorname{IgGs}$ (nIgGs)-treated HUVECs (Supplementary Fig. 4A). In addition, in order to compare the inductive effect of Ang II on HUVECs senescence, cells were treated with Ang II $(1 \mu \mathrm{M})$ and analyzed by western blot. A time-dependent upregulation was also observed. Unlike AT1-AAs, the initiative induction of Ang II was observed at $48 \mathrm{hrs}$ (Supplementary Fig. 4B).

Cellular senescence is characterized by suppressed cell proliferation and permanent cell-cycle arrest. Our results showed that the proliferative activity was reduced in HUVECs treated with AT1-AAs-IgGs as compared with cells in the nIgGs-treated group (Fig. 5D). In addition, the rate of HUVECs arrested in the G0/G1 phase was higher and in the S/M phase was lower in the AT1AAs-IgGs group (Fig. 5E). To examine the effect of AT1AAs-IgGs on HUVECs senescence, senescenceassociated $\beta$-galactosidase (SA $\beta$-gal) staining, a biomarker of cellular senescence, was performed. As shown in Fig. 5F, AT1-AAs-IgGs induced a high positive rate of SA $\beta$-gal, from $2.37 \pm 0.31$ in nIgGs-treated HUVECs to $23.59 \pm 2.45$ in cells treated with $1 \mu \mathrm{M}$ of AT1-AAs-IgGs. Tumour necrosis factor alpha (TNF- $\alpha$ ), interleukin (IL)- 6 and monocyte chemoattractant protein (MCP)-1 are all important inflammatory factors involved in EC senescence. Herein, the mRNA levels of these factors were determined by RT-qPCR analysis. The results showed that AT1-AAs-IgGs increased the transcription of TNF $\alpha$, IL- 6 and MCP-1 drastically. In addition, treatment with AT1-AAs-IgGs enhanced the mRNA levels of intercellular adhesion molecule-1
(ICAM-1), vascular cell adhesion molecule-1 (VCAM-1) and plasminogen activator inhibitor (PAI)-1 (Supplementary Fig. 6). Finally, to determine whether the pro-senescent effect of AT1-AAs-IgGs was AT1 receptor-dependent, HUVECs were pre-incubated with valsartan $(10 \mu \mathrm{M})$. As shown in Fig. 5C-G and Fig. 5, AT1-AAs-IgGs-induced upregulation of senescence markers, retardation of cell proliferation and cell cycle, SA $\beta$-gal-positive rate, as well as reduced phosphorylation of eNOS were almost completely blocked by valsartan. Besides, when cultured HUVECs were incubated with valsartan, the expression of the inflammatory molecules was significantly reduced (Supplementary Fig. 6). All these findings suggested that AT1-AAs-IgGs induced EC senescence, which was most probably through the AT1 receptor pathway.

\section{AT1-AAs from the PAD patients induced HUVECs senescence}

AT1-AAs-IgGs were purified from the AT1-AAspositive PAD patients, while nIgGs were from the nonPAD volunteers. The beating frequency of NRCMs was counted to determine the activity of AT1-AAs-IgGs. As Supplementary Fig. 7 shows, the AT1-AAs-IgGs markedly enhanced the beating frequency of NRCMs and could be inhibited by valsartan. Senescent markers expression and SA $\beta$-gal staining were performed to examine the effect of AT1-AAs-IgGs on HUVECs senescence. As shown in Fig. 6, AT1-AAs-IgGs induced increased expression of p53, p21 and p16 ${ }^{\mathrm{INK} 4 \mathrm{a}}$ as well as a high positive rate of SA $\beta$-gal. The pro-senescent effect was mostly blocked by treatment with valsartan.

\section{DISCUSSION}

The main findings of our research are (1) a high serum AT1-AAs level was closely associated with PAD; (2) long-term exposure to AT1-AAs could induce vascular aging and EC senescence in vivo; (3) AT1-AAs-IgGs induced EC senescence in an AT1 receptor-dependent pathway. We demonstrate for the first time that excess activation of AT1 receptor by its agonist autoantibody 
contribute to vascular aging and EC senescence, which provides important insights into the mechanism underlying vascular aging and $\mathrm{EC}$ senescence.

CVD are the leading cause of death worldwide. Aging of the large conduit arteries is a major cause of morbidity and mortality in the aging population. The aged arteries, characterized by complex structural and functional changes, are prone to hypertension, arterial calcification, atherosclerosis and increased stiffness. Autoantibodies against AT1 receptor, namely AT1-AAs, were first discovered in the serum of patients with pre-eclampsia [13]. Many studies have found that AT1-AAs exhibited an agonist-like activity like Ang II [24, 25]. In recent years, accumulating evidence suggested that AT1-AAs were involved in the pathogenesis of vascular injury [18, 26]. Besides, it has been shown that the AT1-AAs levels were correlated with aging phenotypes [20]. Based on the above, the question arises as to whether high serum levels of AT1-AAs play roles in accelerating vascular aging on the basis of increased cellular senescence. Our data showed that AT1-AAs provoked premature aging of the aortas and accelerated EC senescence. This finding provides a new clue about the association of elevated serum AT1-AAs level with CVD, and AT1-AAs may prove to be a hallmark hazard of endothelial senescence and atherogenesis.

A series of epidemiological studies have demonstrated that PAD is prevalent in the elderly. In clinical practice, PAD can be detected by the measurement of ankle-brachial index (ABI) and is diagnosed by the presence of an ABI < 0.9 [27]. The PAD is not just a disease of the peripheral arteries, but also is an indication of high probability of generalized vascular atherosclerosis [22] and other atherosclerotic diseases such as coronary artery disease and stroke [28]. This study examined the relationship between serum AT1-AAs level and PAD. The results revealed that in the PAD group, the positive rate of serum AT1-AAs was higher, and the levels were prevalently high, compared with the non-PAD volunteers. In comparison to non-PAD volunteers, PAD patients showed a high prevalence of common risk factors, including smoking history, hypertension, diabetes and dyslipidemia (Table 1, 2). Nevertheless, after adjustment for these factors, the serum AT1-AAs level was significantly associated with PAD (Table 3 ). These results suggested that serum AT1-AAs were closely related to vascular aging-related diseases.

Active immunization is a classic method for studying a variety of antibodies, which has been proven to be able to elicit autoantibodies and cause a similar syndrome as passive transfer of autoantibodies [19, 29]. Our previous research has demonstrated that AT1-AAs from the actively immunized rats and preeclamptic patients showed similar biological activity [18]. Our results showed that there were constant high levels of circulating antibodies throughout the study period in all rats of the immunized group, but not in the control rats (Fig. 2A). Therefore, antibodies in the current study could be considered as AT1-AAs.

To explore the role of AT1-AAs in vascular aging, we examined the time course of multiple noninvasive and invasive arterial physiological parameters and structural changes of the arteries in rats. Stiffening of large elastic arteries has emerged as a major independent risk factor for age-associated CVD [4]. In our study, AT1-AAs reduced carotid compliance with increased PWV. Besides, we demonstrated that endothelium-dependent vasodilatation of the immunized arteries was reduced. Actually, both of the above characteristics are thought to be key functional indicators of vascular aging and significantly contribute to CVD in the elderly [30]. eNOS, through generating the gasotransmitter nitric oxide (NO), plays an essential role in the regulation of endothelial function. Thus, eNOS acts as a master regulator of vascular tone and homeostasis. Dysregulation of the eNOS-NO axis induces endothelial dysfunction [31]. Herein, we found reduced eNOS phosphorylation of the aortas in the immunized rats (Supplementary Fig. 2). This suggests AT1-AAs might inhibit the activity of eNOS, but the mechanisms need to be further explored. During the process of immunization, we found that SBP began increasing at the 2 nd week and sustained to the 4 th week. However, the SBP showed no significant difference between the vehicle and immunized groups from the 6th week. Consistent with our findings, SBP was unchanged in the study by Wang et al [26]. It might be because that blood pressure in the immunized rats was adjusted to normal by other antihypertensive mechanisms, or due to decreased cardiac function by AT1-AAs as previous study confirmed [32].

Endothelial damage has been recognized as the key step in the pathophysiology of diverse cardiovascular abnormalities associated with aging. Vascular cell senescence, especially EC, is involved in vascular aging [33]. EC senescence has been recognized to be linked to aging-related endothelial dysfunction and atherosclerosis [34]. Thus, the slowing of EC senescence is emerging as an exciting possibility for controlling vascular diseases. In the present study, we demonstrated for the first time that AT1-AAs could induce vascular aging, which is considered the early event in the development of atherosclerosis. Especially, AT1-AAs exposure caused endothelial dysfunction and endothelial senescence in vivo. Besides, our in vitro data suggested that AT1-AAsIgGs extracted from the serum of immunized rats induced EC senescence, even in the absence of other environmental injury stimuli, and could be blocked by the AT1 receptor blocker. Our findings that AT1-AAs treatment caused HUVECs senescence in vitro is 
consistent with spontaneous vascular aging in $\mathrm{AT}_{1} \mathrm{R}-\mathrm{EC}_{\mathrm{II}}{ }^{-}$ immunized rats. Cellular senescence was initially described as the limited ability of somatic cells to divide when cultured in vitro, which was termed as replicative senescence [35]. More recently, it has been increasingly realized that EC senescence can also be induced by a number of stress stimuli and thus leads to stress-induced premature senescence (SIPS) [36]. Compared with the replicative senescence, usually accompanied by telomere shortening, SIPS seems to be particularly relevant to vascular aging, in which the vessel wall-resident ECs may not be able to replicate sufficiently [37].

Numerous studies have demonstrated that activation of the AT1 receptor plays vital roles in regulating the aging processes of the vascular system [38]. Recently discovered autoantibodies against AT1 receptor (AT1AAs) might provide some new ideas about the complicated pathological process. The main mechanism of the agonistic effect involves the molecular mimicry theory, where AT1-AAs specifically bind to the second loop of the AT1 receptor and activate it [13]. Accumulating evidence indicates that AT1-AAs induce several senescence-associated signaling mechanisms, including reactive oxygen species (ROS) production, inflammatory response and NF- $\mathrm{KB}$ activation by activating the reduced form of nicotinamide adenine dinucleotide phosphate (NADPH) oxidase (Nox) [16, 39]. Oxidative stress has been considered a key driver of the aging process, and subsequent studies have revealed the link between oxidative stress and senescence [40]. It has been shown that ROS contributes to endothelial senescence by decreasing NO production, promoting inflammation and perturbing ECs functions [41, 42]. Nox, which produces ROS, is pivotal to EC senescence and the pathophysiology of various vascular diseases [43, 44]. Inflammation is recognized as a primary pathological mechanism of aging-related endothelial dysfunction and aging of arteries [45]. The serum concentrations of inflammatory proteins increase with age, including proinflammatory cytokines such as TNF- $\alpha$, IL- 6 and MCP-1. Therefore, the upregulation of these cytokines with AT1AAs treatment reflects a chronic pro-inflammatory state correlated with aging. Such a condition is attributable to $\mathrm{NF}-\kappa \mathrm{B}$ activation [46]. Indeed, NF- $\kappa \mathrm{B}$ has been reported to be activated and promote gene transcription of proinflammatory cytokines after AT1-AAs treatment [17]. Moreover, NF- $\mathrm{\kappa B}$ gene expression may result in increased levels of ICAM-1 and VCAM-1. AT1-AAs may also contribute to the surface adhesion molecule expression and tissue factor production [47, 48]. In addition to the above possibilities, AT1-AAs might induce EC senescence via the activation of small G-proetin Ras, mitogen-activated protein kinases and other transcription factors such as activator protein (AP)-1, all of which are the signaling cascades downstream of AT1 receptor activation [49].

Under the conditions of the same total doses, the AT1-AAs-induced EC senescence occurred later than that of Ang II (Supplementary Fig. 4B), indicating a relative weaker inductive effect of AT1-AAs. Nevertheless, the specific reasons for this need to be further explored. Unlike the natural ligands, the agonistic autoantibodies are able to promote a sustained receptor activation without the customary desensitization $[50,51]$. Thus, the detrimental effects of AT1-AAs may be attributed to the excessive AT1 receptor activation. Beyond that, a previous study has shown that AT1-AAs might increase the sensitivity of Ang II in ECs [52].

In summary, our findings show a novel pathological factor contributing to vascular aging, especially EC senescence. The increasing level of serum AT1-AAs should be considered as a clinical assessment factor in the deterioration of vascular-aging related diseases.

\section{Acknowledgements}

This research was supported by funding from the Major Program of National Natural Science Foundation of China (91539205, 2016), Natural Sciences Foundation of China (31771267, 2018), Beijing Natural Science Foundation Program and Scientific Research Key Program of Beijing Municipal Commission of Education (KZ201810025039, 2018), China Postdoctoral Science Foundation (2017M610945).

\section{Conflict of interest}

The authors declare no conflict of interest.

\section{Supplementary Materials}

The Supplemenantry data can be found online at: www.aginganddisease.org/EN/10.14336/AD.2018.0919.

\section{References}

[1] North BJ, Sinclair DA (2012). The intersection between aging and cardiovascular disease. Circ Res, 110:10971108 .

[2] Sindler AL, Fleenor BS, Calvert JW, Marshall KD, Zigler ML, Lefer DJ, et al. (2011). Nitrite supplementation reverses vascular endothelial dysfunction and large elastic artery stiffness with aging. Aging Cell, 10:429-437.

[3] Harvey A, Montezano AC, Touyz RM (2015). Vascular biology of ageing-Implications in hypertension. J Mol Cell Cardiol, 83:112-121.

[4] Lakatta EG, Levy D (2003). Arterial and cardiac aging: major shareholders in cardiovascular disease 
enterprises: Part I: aging arteries: a "set up" for vascular disease. Circulation, 107:139-146.

[5] Fitch RM, Vergona R, Sullivan ME, Wang YX (2001). Nitric oxide synthase inhibition increases aortic stiffness measured by pulse wave velocity in rats. Cardiovasc Res, 51:351-358.

[6] Tian XL, Li Y (2014). Endothelial cell senescence and age-related vascular diseases. J Genet Genomics, 41:485-495.

[7] Minamino T, Miyauchi H, Yoshida T, Ishida Y, Yoshida H, Komuro I (2002). Endothelial cell senescence in human atherosclerosis: role of telomere in endothelial dysfunction. Circulation, 105:1541-1544.

[8] Te Riet L, van Esch JH, Roks AJ, van den Meiracker AH, Danser AH (2015). Hypertension: reninangiotensin-aldosterone system alterations. Circ Res, 116:960-975.

[9] Ng K, Hildreth CM, Avolio AP, Phillips JK (2011). Angiotensin-converting enzyme inhibitor limits pulsewave velocity and aortic calcification in a rat model of cystic renal disease. Am J Physiol Renal Physiol, 301:F959-966.

[10] Shan H, Zhang S, Li X, Yu K, Zhao X, Chen X, et al. (2014). Valsartan ameliorates ageing-induced aorta degeneration via angiotensin II type 1 receptormediated ERK activity. J Cell Mol Med, 18:1071-1080.

[11] Unal H, Karnik SS (2014). Constitutive activity in the angiotensin II type 1 receptor: discovery and applications. Adv Pharmacol, 70:155-174.

[12] Li T, Yu B, Liu Z, Li J, Ma M, Wang Y, et al. (2018). Homocysteine directly interacts and activates the angiotensin II type I receptor to aggravate vascular injury. Nat Commun, 9:11.

[13] Wallukat G, Homuth V, Fischer T, Lindschau C, Horstkamp B, Jupner A, et al. (1999). Patients with preeclampsia develop agonistic autoantibodies against the angiotensin AT1 receptor. J Clin Invest, 103:945952.

[14] Fu ML, Herlitz H, Schulze W, Wallukat G, Micke P, Eftekhari P, et al. (2000). Autoantibodies against the angiotensin receptor (AT1) in patients with hypertension. J Hypertens, 18:945-953.

[15] Zhu F, Sun YX, Liao YH, Wei YM, Chen M, Wang M, et al. (2008). Agonistic AT(1) receptor autoantibody increases in serum of patients with refractory hypertension and improves $\mathrm{Ca}(2+)$ mobilization in cultured rat vascular smooth muscle cells. Cell Mol Immunol, 5:209-217.

[16] Dragun D, Muller DN, Brasen JH, Fritsche L, Nieminen-Kelha M, Dechend R, et al. (2005). Angiotensin II type 1-receptor activating antibodies in renal-allograft rejection. N Engl J Med, 352:558-569.

[17] Li W, Li Z, Chen Y, Li S, Lv Y, Zhou W, et al. (2014). Autoantibodies targeting AT1 receptor from patients with acute coronary syndrome upregulate proinflammatory cytokines expression in endothelial cells involving NF-kappaB pathway. J Immunol Res, 2014:342693.

[18] Zhang SL, Du YH, Wang J, Yang LH, Yang XL, Zheng RH, et al. (2010). Endothelial dysfunction induced by antibodies against angiotensin AT1 receptor in immunized rats. Acta Pharmacol Sin, 31:1381-1388.

[19] Li W, Chen Y, Li S, Guo X, Zhou W, Zeng Q, et al. (2014). Agonistic antibody to angiotensin II type 1 receptor accelerates atherosclerosis in ApoE-/- mice. Am J Transl Res, 6:678-690.

[20] Abadir PM, Jain A, Powell LJ, Xue QL, Tian J, Hamilton RG, et al. (2017). Discovery and Validation of Agonistic Angiotensin Receptor Autoantibodies as Biomarkers of Adverse Outcomes. Circulation, 135:449-459.

[21] Fowkes FG, Rudan D, Rudan I, Aboyans V, Denenberg JO, McDermott MM, et al. (2013). Comparison of global estimates of prevalence and risk factors for peripheral artery disease in 2000 and 2010: a systematic review and analysis. Lancet, 382:1329-1340.

[22] Scandale G, Dimitrov G, Recchia M, Carzaniga G, Minola M, Perilli E, et al. (2018). Arterial stiffness and subendocardial viability ratio in patients with peripheral arterial disease. J Clin Hypertens (Greenwich), 20:478484.

[23] Vlachopoulos C, Aznaouridis K, Stefanadis C (2010). Prediction of cardiovascular events and all-cause mortality with arterial stiffness: a systematic review and meta-analysis. J Am Coll Cardiol, 55:1318-1327.

[24] Walther T, Stepan H (2007). Agonist autoantibodies against the angiotensin AT1 receptor in renal and hypertensive disorders. Curr Hypertens Rep, 9:128-132. [25] Okruhlicova L, Morwinski R, Schulze W, Bartel S, Weismann P, Tribulova N, et al. (2007). Autoantibodies against G-protein-coupled receptors modulate heart mast cells. Cell Mol Immunol, 4:127-133.

[26] Wang B, Liao YH, Zhou Z, Li L, Wei F, Wang M, et al. (2005). Arterial structural changes in rats immunized by AT1-receptor peptide. Heart Vessels, 20:153-158.

[27] Hirsch AT, Haskal ZJ, Hertzer NR, Bakal CW, Creager MA, Halperin JL, et al. (2006). ACC/AHA 2005 Practice Guidelines for the management of patients with peripheral arterial disease (lower extremity, renal, mesenteric, and abdominal aortic): a collaborative report from the American Association for Vascular Surgery/Society for Vascular Surgery, Society for Cardiovascular Angiography and Interventions, Society for Vascular Medicine and Biology, Society of Interventional Radiology, and the ACC/AHA Task Force on Practice Guidelines (Writing Committee to Develop Guidelines for the Management of Patients With Peripheral Arterial Disease): endorsed by the American Association of Cardiovascular and Pulmonary Rehabilitation; National Heart, Lung, and Blood Institute; Society for Vascular Nursing; TransAtlantic Inter-Society Consensus; and Vascular Disease Foundation. Circulation, 113:e463-654.

[28] Wakabayashi I, Sotoda Y, Hirooka S, Orita H (2015). Association between cardiometabolic index and atherosclerotic progression in patients with peripheral arterial disease. Clin Chim Acta, 446:231-236.

[29] Fu ML, Leung PS, Wallukat G, Bergstrom G, Fu H, Schulze W, et al. (1999). Agonist-like activity of antibodies to angiotensin II receptor subtype 1 (AT1) 
from rats immunized with AT1 receptor peptide. Blood Press, 8:317-324.

[30] Shirwany NA, Zou MH (2010). Arterial stiffness: a brief review. Acta Pharmacol Sin, 31:1267-1276.

[31] Tousoulis D, Kampoli AM, Tentolouris C, Papageorgiou N, Stefanadis C (2012). The role of nitric oxide on endothelial function. Curr Vasc Pharmacol, 10:4-18.

[32] Jin Z, Wang J, Zhang W, Zhang G, Jiao X, Zhi J (2011). Changes in cardiac structure and function in rats immunized by angiotensin type 1 receptor peptides. Acta Biochim Biophys Sin (Shanghai), 43:970-976.

[33] Yin H, Pickering JG (2016). Cellular Senescence and Vascular Disease: Novel Routes to Better Understanding and Therapy. Can J Cardiol, 32:612-623.

[34] Minamino T, Komuro I (2007). Vascular cell senescence: contribution to atherosclerosis. Circ Res, 100:15-26.

[35] Hayflick L (1965). The Limited In Vitro Lifetime Of Human Diploid Cell Strains. Exp Cell Res, 37:614-636.

[36] Chen J, Patschan S, Goligorsky MS (2008). Stressinduced premature senescence of endothelial cells. J Nephrol, 21:337-344.

[37] Marcelo KL, Goldie LC, Hirschi KK (2013). Regulation of endothelial cell differentiation and specification. Circ Res, 112:1272-1287.

[38] Griendling KK, Sorescu D, Ushio-Fukai M (2000). $\mathrm{NAD}(\mathrm{P}) \mathrm{H}$ oxidase: role in cardiovascular biology and disease. Circ Res, 86:494-501.

[39] Dechend R, Viedt C, Muller DN, Ugele B, Brandes RP, Wallukat G, et al. (2003). AT1 receptor agonistic antibodies from preeclamptic patients stimulate NADPH oxidase. Circulation, 107:1632-1639.

[40] Harman D (1981). The aging process. Proc Natl Acad Sci U S A, 78:7124-7128.

[41] Gryglewski RJ, Palmer RM, Moncada S (1986). Superoxide anion is involved in the breakdown of endothelium-derived vascular relaxing factor. Nature, 320:454-456.

[42] Marui N, Offermann MK, Swerlick R, Kunsch C, Rosen CA, Ahmad M, et al. (1993). Vascular cell adhesion molecule-1 (VCAM-1) gene transcription and expression are regulated through an antioxidant- sensitive mechanism in human vascular endothelial cells. J Clin Invest, 92:1866-1874.

[43] Meijles DN, Sahoo S, Al Ghouleh I, Amaral JH, BienesMartinez R, Knupp HE, et al. (2017). The matricellular protein TSP1 promotes human and mouse endothelial cell senescence through CD47 and Nox1. Sci Signal, 10.

[44] Gray SP, Jandeleit-Dahm KA (2015). The role of NADPH oxidase in vascular disease--hypertension, atherosclerosis \& stroke. Curr Pharm Des, 21:59335944.

[45] Dinh QN, Drummond GR, Sobey CG, Chrissobolis S (2014). Roles of inflammation, oxidative stress, and vascular dysfunction in hypertension. Biomed Res Int, 2014:406960.

[46] Csiszar A, Wang M, Lakatta EG, Ungvari Z (2008). Inflammation and endothelial dysfunction during aging: role of NF-kappaB. J Appl Physiol (1985), 105:13331341.

[47] Haller H, Ziegler EM, Homuth V, Drab M, Eichhorn J, Nagy Z, et al. (1997). Endothelial adhesion molecules and leukocyte integrins in preeclamptic patients. Hypertension, 29:291-296.

[48] Dechend R, Homuth V, Wallukat G, Kreuzer J, Park JK, Theuer J, et al. (2000). AT(1) receptor agonistic antibodies from preeclamptic patients cause vascular cells to express tissue factor. Circulation, 101:23822387.

[49] Min LJ, Mogi M, Iwai M, Horiuchi M (2009). Signaling mechanisms of angiotensin II in regulating vascular senescence. Ageing Res Rev, 8:113-121.

[50] Xia Y, Kellems RE (2013). Angiotensin receptor agonistic autoantibodies and hypertension: preeclampsia and beyond. Circ Res, 113:78-87.

[51] Zhang S, Zheng R, Yang L, Zhang X, Zuo L, Yang X, et al. (2013). Angiotensin type 1 receptor autoantibody from preeclamptic patients induces human fetoplacental vasoconstriction. J Cell Physiol, 228:142148.

[52] Wenzel K, Rajakumar A, Haase H, Geusens N, Hubner $\mathrm{N}$, Schulz H, et al. (2011). Angiotensin II type 1 receptor antibodies and increased angiotensin II sensitivity in pregnant rats. Hypertension, 58:77-84. 